\title{
Boundary shape and Casimir energy
}

\author{
H Ahmedov ${ }^{1}$ and I H Duru ${ }^{2}$ \\ ${ }^{1}$ Feza Gürsey Institute, P. K. 6 Çengelköy, 34684 Istanbul, Turkey \\ ${ }^{2}$ Izmir Institute of Technology, 35430 Izmir, Turkey \\ E-mail: hagi@gursey.gov.tr and ihduru@galois.iyte.edu.tr
}

Received 24 October 2008, in final form 15 December 2008

Published 17 February 2009

Online at stacks.iop.org/JPhysA/42/115401

\begin{abstract}
Casimir energy changes are investigated for geometries obtained by small but arbitrary deformations of a given geometry for which the vacuum energy is already known for the massless scalar field. As a specific case, deformation of a spherical shell is studied. From the deformation of the sphere we show that the Casimir energy is a decreasing function of the surface-to-volume ratio. The decreasing rate is higher for less smooth deformations.
\end{abstract}

PACS numbers: $03.65 .-\mathrm{w}, 03.70 .+\mathrm{k}$

\section{Introduction}

Casimir energies are known for several cavities in several spatial dimensions for electromagnetic or massless scalar fields. Exact Casimir energy calculations are available for rectangular prisms [1], for spherical shell [2], for a cylindrical region [3], for a pyramidal cavity and for a conical cavity [4]. All these geometries have definite boundary wall shapes. For example, the prisms are all with right angular wedges, the sphere is the perfect one; and, the pyramid and the cone are of very special types. The obvious reason of these restrictions is the fact that these are the regions for which one can calculate the exact field modes with the required boundary conditions. Limited number of examples (all with rigid walls) do of course not give much hint about the dependence of the Casimir energy on the shapes of the regions.

There are to our knowledge two approaches to dealing with rather arbitrary geometries. The first is the proximity force approximation [5]. It is applicable to two body systems which are close to each other. It employs the parallel plate modes in every cylindrical region of infinitesimal base between the bodies and then integrates over these regions [6]. In this sense the so-called pointwise summation methods too can be considered as proximity force approximation [7]. The second approach is the one called multiple scattering expansion [8] which relies on the connection between closed classical paths and Casimir energy [9]. It formulates the vacuum energy for the electromagnetic field in terms of the successive scatterings from the conducting boundaries [10]. In this approach between two 
successive scatterings free Green function is employed. The method enables one to investigate the connections between the divergencies and the geometrical details of the boundaries. Employment of the free Green functions however from one scattering to the next one is not of much practical value in compact space regions: specially for more curved boundaries one may need to consider large number of scatterings to approximate the exact Green function. On the other hand, it may be more reasonable to employ the exact Green function (if it is already known) of the compact region (instead of the free one) if the region under consideration is sufficiently close to the original region. Variations around the exactly solvable geometries may give some hint about the dependence of the vacuum energies on the shape of the boundaries. Note that in principle the path integral approach in its basic formulation can be adopted to the perturbation around any Green function. However its development has been only performed for two body systems as expansion around the Green function of two plates [11].

In present work, we try to investigate the shape dependence of the Casimir energy for massless scalar field by calculating the effect of the small but arbitrary deformations of a given geometry for which we already know the vacuum energies. We use the Green function to formulate the perturbation theory around the exact solution of the region with boundary $S$. Suppose $G_{\omega}^{S}$ is the exact Green function for the massless scalar field confined to the region in $S$, and $\beta$ is the small deformation of $S$. Converting a boundary problem into an integral equation we arrive at the perturbation series

$$
G_{\omega}^{\tilde{S}}=G_{\omega}^{S}+\beta G_{\omega}^{1 S}+\beta^{2} G_{\omega}^{2 S}+\cdots
$$

where $G_{\omega}^{j S}$ is the correction to the original Green function $G_{\omega}^{S}$ resulting from the $j$ times reflections from the deformed boundary $\tilde{S}$. One reflection gives information about the size of the new boundary. To get information about the shape dependence we need to take into account at least two reflections.

Having in hand the Green function we can construct the zeta function which is a useful tool in Casimir energy calculation. The zeta function of the system can be expressed in terms of the heat kernel coefficients which are functionals of the geometrical invariants of the boundaries [12]. For massless fields which are the only fields for which the Casimir energy is meaningful, there is no unique way of getting rid of the infinities if the heat kernel coefficient $a_{2}$ of the zeta function expansion is not zero [13]. The situation can be improved if one considers the whole space for when one sums the zeta functions of the in and out regions $a_{2}$ coefficients cancel each other. Of course for such cancelation the boundaries should be free of sharp corners.

In the following section we briefly review the zeta function approach to the vacuum energy calculations and the regularization scheme which we employ in our work.

In section 3 we present the general formulation of the Casimir energy contribution of small deformations of the boundaries.

In section 4 deformation of the sphere is discussed.

In section 5 we analyze the dependence of the energy on the shape of the boundary.

Details of the involved calculations are given in the appendices.

\section{A brief review of the zeta function method}

Formally the calculation of the Casimir energy is reduced to a treatment of a sum over all one-particle energy eigenvalues

$$
E=\frac{1}{2} \sum_{\lambda \in \Lambda} \sqrt{E_{\lambda}} .
$$


This sum is divergent and regularization is needed. For the scalar field confined in a compact three-dimensional region with the Dirichlet boundary condition the zeta function

$$
\zeta(z)=\sum_{n=1}^{\infty} E_{n}^{-z}
$$

is well defined by the Weyl theorem for $\operatorname{Re} z>3 / 2$ [14]. By the analytic continuation it is possible to define this function on the whole complex plane. This may be done by using the representation

$$
\zeta(z)=\frac{1}{\Gamma(z)} \int_{0}^{\infty} \mathrm{d} t t^{z-1} K(t)
$$

with the heat kernel

$$
K(t)=\sum_{n=1}^{\infty} \mathrm{e}^{-t E_{n}}
$$

For $t \rightarrow \infty$ the integral is well behaved. Possible poles arise from $t \rightarrow 0$ behavior of the heat kernel [12]

$$
K(t) \sim \sum_{n=0,1 / 2,1, \ldots}^{\infty} a_{n} t^{n-3 / 2}
$$

Splitting the integral as $\int_{0}^{1} \mathrm{~d} t+\int_{1}^{\infty} \mathrm{d} t$ we arrive at

$$
\left.\operatorname{Re} s(\zeta(z) \Gamma(z))\right|_{z=3 / 2-n}=a_{n}
$$

where $a_{n}$ are heat kernel coefficients which depend on the geometry of boundary which confines the scalar field. When the coefficient $a_{2}$ is nonzero the value of the zeta function at $z=-1 / 2$ which defines the Casimir energy becomes infinite. For massless scalar field there is no renormalization condition to get rid of this pole in a unique way [13]. One needs additional considerations to apply the zeta function method to the calculation of the vacuum energy. The extrinsic curvature of the sphere will have opposite sign when viewed from inside or outside. $a_{2}$ heat-kernel coefficient depends on an odd power of extrinsic curvature. Two of them add to cancel each other when we approach the surface both from in and out regions of the ball. This does not hold only for the spherical shell but is a general property for boundaries of an arbitrary shape. This cancelation of poles occurs only for infinitely thin boundaries. Once a finite thickness is introduced the absolute value of the extrinsic curvature at the inner and outer sides of the boundary is different and divergencies do not cancel each other.

In the present work, we try to investigate the shape dependence of the Casimir energy for the massless scalar field by calculating the effect of the small but arbitrary smooth deformations of the boundary of given regions for which we already know the vacuum energies. We restrict our attention to the deformations of the spherical shell. Using the scattering theory in the spherical coordinates one arrives at the zeta functions inside

$$
\zeta_{\text {in }}(z)=\frac{\sin \pi z}{\pi} \sum_{l=0}^{\infty}(2 l+1) \int_{0}^{\infty} \mathrm{d} \omega \omega^{-2 z} \frac{\mathrm{d}}{\mathrm{d} \omega} \ln \left(\omega^{-l-1 / 2} I_{l+1 / 2}(\omega)\right)
$$

and outside the ball $[13,14]$

$$
\zeta_{\text {out }}(z)=\frac{\sin \pi z}{\pi} \sum_{l=0}^{\infty}(2 l+1) \int_{0}^{\infty} \mathrm{d} \omega \omega^{-2 z} \frac{\mathrm{d}}{\mathrm{d} \omega} \ln \left(\omega^{l+1 / 2} K_{l+1 / 2}(\omega)\right) .
$$

The zeta function in the whole space

$$
\zeta(z)=\frac{1}{2}\left(\zeta_{\text {in }}(z)+\zeta_{\text {out }}(z)\right)
$$


or

$$
\zeta(z)=\frac{\sin \pi z}{\pi} \sum_{l=0}^{\infty}(l+1 / 2) \int_{0}^{\infty} \mathrm{d} \omega \omega^{-2 z} \frac{\mathrm{d}}{\mathrm{d} \omega} \ln \left(I_{l+1 / 2}(\omega) K_{l+1 / 2}(\omega)\right)
$$

is well defined at $z=-1 / 2$. To find this value we use the uniform asymptotic expansions for Bessel functions [15]

$$
K_{v}(v x)=\sqrt{\frac{\pi}{2 v}} \frac{\mathrm{e}^{-v \eta}}{\left(1+x^{2}\right)^{1 / 4}}\left(1+\sum_{k=1}^{\infty}(-)^{k} \frac{u_{k}(t)}{v^{k}}\right)
$$

and

$$
I_{\nu}(v x)=\frac{1}{\sqrt{2 \pi v}} \frac{\mathrm{e}^{\nu \eta}}{\left(1+x^{2}\right)^{1 / 4}}\left(1+\sum_{k=1}^{\infty} \frac{u_{k}(t)}{v^{k}}\right)
$$

where

$$
t=\frac{1}{\sqrt{1+x^{2}}}, \quad \eta=\sqrt{1+x^{2}}+\ln \frac{x}{1+\sqrt{1+x^{2}}}
$$

and coefficients $u_{k}(t)$ satisfy the recurrence relation

$$
u_{k+1}(t)=\frac{1}{2} t^{2}\left(1-t^{2}\right) u_{k}^{\prime}(t)+\frac{1}{8} \int_{0}^{t} \mathrm{~d} \tau\left(1-5 \tau^{2}\right) u_{k}(\tau)
$$

with the initial condition $u_{0}(t)=1$. The uniform asymptotic expansions imply

$$
\ln I_{v}(v x)=\sum_{-1}^{\infty} \frac{X_{k}(t)}{v^{k}}
$$

and

$$
\ln K_{v}(v x)=\sum_{-1}^{\infty}(-)^{k} \frac{X_{k}(t)}{v^{k}}
$$

where the first four terms of $X_{n}(t)$ s are

$$
X_{-1}=\frac{1}{t}+\ln \frac{t}{1+t}, \quad X_{0}=\frac{1}{2} \ln t, \quad X_{1}=\frac{t}{8}-\frac{5 t^{3}}{24}, \quad X_{2}=\frac{t^{2}}{16}-\frac{3 t^{4}}{8}+\frac{5 t^{6}}{16} .
$$

The zeta function in the whole space becomes

$$
\zeta(-1 / 2)=\frac{2}{\pi} \sum_{m=0}^{\infty} \zeta_{0}(2 m-2) \int_{0}^{\infty} \mathrm{d} x X_{2 m}(t)
$$

where

$$
\zeta_{0}(z)=\sum_{n=0}^{\infty} \frac{1}{(1 / 2+n)^{z}}
$$

is the zeta function which vanishes for $z=0$ and $z=-2$ [17]. The vacuum energy for the massless scalar field in the spherical shell of radius $R$ is [16]

$$
E_{\mathrm{sph}}=\frac{1}{2 R} \zeta(-1 / 2) \simeq \frac{\alpha}{R}, \quad \alpha \simeq 0,003 .
$$




\section{Contribution of small boundary deformations to the vacuum energy}

In this section we use the Green function representation of the zeta function for the massless scalar field in the three-dimensional space vanishing on a surface $S$

$$
\zeta^{S}(z)=\frac{\sin (\pi z)}{\pi} \int_{0}^{\infty} \mathrm{d} \omega \omega^{-2 z+1} \int_{R^{3}} \mathrm{~d}^{3} \vec{x} G_{\omega}^{S}(\vec{x}, \vec{x})
$$

where

$$
G_{\omega}^{S}(\vec{x}, \vec{y})= \begin{cases}G_{\omega}^{\mathrm{in} S}(\vec{x}, \vec{y}), & \vec{x}, \vec{y} \in \Omega_{\mathrm{in}} \\ G_{\omega}^{\mathrm{out} S}(\vec{x}, \vec{y}), & \vec{x}, \vec{y} \in \Omega_{\mathrm{out}}\end{cases}
$$

is the Green function in $R^{3}$ satisfying the boundary problem

$$
\left(-\Delta+\omega^{2}\right) G_{\omega}^{S}(\vec{x}, \vec{y})=\delta(\vec{x}-\vec{y}), \quad G_{\omega}^{S}(\vec{x}, \vec{y})=0, \quad \vec{x} \in S .
$$

Here $G_{\omega}^{\text {in } S}\left(G_{\omega}^{\text {out } S}\right)$ is the Green function in the in-region $\Omega_{\text {in }}$ (the out-region $\Omega_{\text {out }}$ ). To have a well-defined integral over $R^{3}$ in (21) one considers a ball of radius $L$ and then let it go to infinite. Divergent terms in powers of $\mathrm{L}$ will correspond to the infinite vacuum oscillations of the free Minkowski space. The variation

$$
\delta \zeta(z)=\zeta^{\tilde{S}}(z)-\zeta^{S}(z)
$$

resulting from the deformation of the boundary $S$ is then given by

$$
\delta \zeta(z)=\frac{\sin (\pi z)}{\pi} \int_{0}^{\infty} \mathrm{d} \omega \omega^{-2 z+1} \int_{R^{3}} \mathrm{~d}^{3} \vec{x} \delta G_{\omega}(\vec{x}, \vec{x})
$$

where

$$
\delta G_{\omega}(\vec{x}, \vec{y})=G_{\omega}^{\tilde{S}}(\vec{x}, \vec{y})-G_{\omega}^{S}(\vec{x}, \vec{y})
$$

Due to (23) it satisfies the wave equation

$$
\left(-\Delta+\omega^{2}\right) \delta G_{\omega}(\vec{x}, \vec{y})=0
$$

and the boundary condition

$$
\delta G_{\omega}(\vec{x}, \vec{y})=-G_{\omega}^{S}(\vec{x}, \vec{y}), \quad \vec{x} \in \tilde{S} .
$$

The above boundary problem is equivalent to the integral equation [18]

$$
G_{\omega}^{\tilde{S}}(\vec{x}, \vec{y})=G_{\omega}^{S}(\vec{x}, \vec{y})-\int_{\tilde{S}} \mathrm{~d} \tilde{s} \frac{\partial G_{\omega}^{\tilde{S}}(\vec{x}, \vec{v})}{\partial m(\vec{v})} G_{\omega}^{S}(\vec{v}, \vec{y})
$$

where

$$
\frac{\partial}{\partial m(\vec{v})}=\vec{m}(\vec{v}) \frac{\partial}{\partial \vec{v}}
$$

is the derivation along the unit vector $\vec{m}(\vec{v})$ normal to the wall $\tilde{S}$ at a point $\vec{v}$. In the parametric representation $\vec{v}=\vec{v}(\tau), \tau=\left(\tau^{1}, \tau^{2}\right)$ the integration measure on $\tilde{S}$ is

$$
\mathrm{d} \tilde{s}=\sqrt{|\tilde{g}|} \mathrm{d}^{2} \tau
$$

where $|\tilde{g}|$ is the determinant of the induced metric

$$
\tilde{g}_{a b}=\left(\frac{\partial \vec{v}}{\partial \tau^{a}}, \frac{\partial \vec{v}}{\partial \tau^{b}}\right)
$$

with $(\cdot, \cdot)$ being the scalar product in the three-dimensional space. 
The solution of the integral equation (29) up to the second order (which we can also interpret as the second reflection) is

$$
\begin{aligned}
G_{\omega}^{\tilde{S}}(\vec{x}, \vec{y})= & G_{\omega}^{S}(\vec{x}, \vec{y})-\int_{\tilde{S}} \mathrm{~d} \tilde{s} \frac{\partial G_{\omega}^{S}(\vec{x}, \vec{v})}{\partial m(\vec{v})} G_{\omega}^{S}(\vec{v}, \vec{y}) \\
& +\int_{\tilde{S}} \mathrm{~d} \tilde{s} \int_{\tilde{S}} \mathrm{~d} \tilde{s} \frac{\partial G_{\omega}^{S}(\vec{x}, \vec{v})}{\partial m(\vec{v})} \frac{\partial G_{\omega}^{S}\left(\vec{v}, \vec{v}^{\prime}\right)}{\partial m(\vec{v} \prime)} G_{\omega}^{S}\left(\overrightarrow{v^{\prime}}, \vec{y}\right) .
\end{aligned}
$$

The property

$$
\int_{R^{3}} \mathrm{~d}^{3} \vec{x} G_{\omega}^{S}(\vec{z}, \vec{x}) G_{\omega}^{S}\left(\vec{x}, \vec{z}^{\prime}\right)=-\frac{\partial}{\partial \omega^{2}} G_{\omega}^{S}\left(\vec{z}, \overrightarrow{z^{\prime}}\right)
$$

allows us to integrate explicitly the perturbation solution over the three-dimensional spatial space to get

$G_{\omega}^{\tilde{S}}=G_{\omega}^{S}+\frac{1}{2} \frac{\partial}{\partial \omega^{2}} \int_{\tilde{S}} \mathrm{~d} \tilde{s} \frac{\partial G_{\omega}^{S}(\vec{v}, \vec{v})}{\partial m(\vec{v})}-\frac{\partial}{\partial \omega^{2}} \int_{\tilde{S}} \mathrm{~d} \tilde{s} \int_{\tilde{S}} \mathrm{~d} \tilde{s} \frac{\partial G_{\omega}^{S}\left(\overrightarrow{v^{\prime}}, \vec{v}\right)}{\partial m(\vec{v})} \frac{\partial G_{\omega}^{S}\left(\vec{v}, \vec{v}^{\prime}\right)}{\partial m\left(\overrightarrow{v^{\prime}}\right)}$

We consider deformations of the boundary $S$ along the unit vector $\vec{n}(z)$ normal to the surface $S$ at a point $\vec{z}$ :

$$
\vec{v}=\vec{z}-\beta \vec{n}(\vec{z}) f(\vec{z})
$$

where $\beta$ is the dimensionless deformation parameter of the surface $S$. This deformation formula implies

$$
\left.\tilde{g}_{a b}=g_{a b}-\beta\left(\frac{\partial \vec{z}}{\partial \tau^{a}}, \frac{\partial f \vec{n}}{\partial \tau^{b}}\right)+(a \rightarrow b)\right)
$$

where $g_{a b}$ is the metric tensor on $S$. Using $\delta \sqrt{|g|}=1 / 2 \sqrt{|g|} g^{a b} \delta g_{a b}$ we arrive at the variation of the integration measure

$$
\mathrm{d} \tilde{s}=\mathrm{d} s-\beta g^{a b}\left(\frac{\partial \vec{z}}{\partial \tau^{a}}, \frac{\partial(f \vec{n})}{\partial \tau^{b}}\right) \mathrm{d} s .
$$

which together with the Taylor expansion

$$
G_{\omega}^{S}(\vec{x}, \vec{v})=-\beta f(\vec{z}) \frac{\partial G_{\omega}^{S}(\vec{x}, \vec{z})}{\partial n(\vec{z})}+\frac{\beta^{2}}{2} f^{2}(\vec{z}) \frac{\partial^{2} G_{\omega}^{S}(\vec{x}, \vec{z})}{\partial n^{2}(\vec{z})}+\cdots
$$

implies

$$
\begin{gathered}
\delta G_{\omega}=-\frac{\beta}{2} \frac{\partial}{\partial \omega^{2}} \int_{S} \mathrm{~d} s f(\vec{z}) \frac{\partial^{2} G_{\omega}^{S}(\vec{z}, \vec{z})}{\partial n^{2}(\vec{z})}+\frac{\beta^{2}}{2} \frac{\partial}{\partial \omega^{2}} \int_{S} \mathrm{~d} s g^{a b}\left(\frac{\partial \vec{z}}{\partial \tau^{a}}, \frac{\partial(f \vec{n})}{\partial \tau^{b}}\right) f^{2}(\vec{z}) \\
\times \frac{\partial^{2} G_{\omega}^{S}(\vec{z}, \vec{z})}{\partial n^{2}(\vec{z})}+\frac{\beta^{2}}{4} \frac{\partial}{\partial \omega^{2}} \int_{S} \mathrm{~d} s f^{2}(\vec{z}) \frac{\partial^{3} G_{\omega}^{S}(\vec{z}, \vec{z})}{\partial n^{3}(\vec{z})} \\
-\beta^{2} \frac{\partial}{\partial \omega^{2}} \int_{S} \mathrm{~d} s \int_{S} \mathrm{~d} s^{\prime} f(\vec{z}) f\left(\overrightarrow{z^{\prime}}\right)\left(\frac{\partial^{2} G_{\omega}^{S}\left(\overrightarrow{z^{\prime}}, \vec{z}\right)}{\partial n(\vec{z}) \partial n\left(\overrightarrow{z^{\prime}}\right)}\right)^{2} .
\end{gathered}
$$

Up to the second order in $\beta$ the zeta function variation (24) becomes

$$
\delta \zeta(z)=\frac{\sin (\pi z)}{\pi} \int_{0}^{\infty} \mathrm{d} \omega \omega^{-2 z+1} \delta G_{\omega}
$$




\section{Deformation of the spherical shell}

The in and out Green functions for the massless scalar field vanishing on the sphere of the radius $R$ are

$$
\begin{aligned}
G_{\omega}^{\text {in }}\left(r, \vec{n} ; r^{\prime} \vec{n}^{\prime}\right)= & -\frac{1}{4 \pi \sqrt{r r^{\prime}}} \sum_{l=0}^{\infty}(2 l+1) P_{l}\left(\left(\vec{n}, \vec{n}^{\prime}\right)\right) \\
& \times \frac{I_{l+1 / 2}(\omega r)\left(K_{l+1 / 2}(\omega R) I_{l+1 / 2}\left(\omega r^{\prime}\right)-I_{l+1 / 2}(\omega R) K_{l+1 / 2}\left(\omega r^{\prime}\right)\right.}{I_{l+1 / 2}(\omega R)}
\end{aligned}
$$

and

$$
\begin{aligned}
G_{\omega}^{\text {out }}\left(r, \vec{n} ; r^{\prime} \vec{n}^{\prime}\right) & =-\frac{1}{4 \pi \sqrt{r r^{\prime}}} \sum_{l=0}^{\infty}(2 l+1) P_{l}\left(\left(\vec{n}, \vec{n}^{\prime}\right)\right) \\
& \times \frac{\left(K_{l+1 / 2}(\omega R) I_{l+1 / 2}(\omega r)-I_{l+1 / 2}(\omega R) K_{l+1 / 2}(\omega r)\right) K_{l+1 / 2}\left(\omega r^{\prime}\right)}{K_{l+1 / 2}(\omega R)}
\end{aligned}
$$

where $0 \leqslant r \leqslant r^{\prime} \leqslant R, P_{l}(x)$ is the Legendre polynomial and $\vec{n}$ is the unit vector normal to the sphere (see appendix B). The derivative normal to the sphere is $\frac{\partial}{\partial n(\vec{z})}=\frac{\partial}{\partial r}$. We have the derivatives of the following type:

$$
\left.\frac{\partial^{2} G_{\omega}^{S^{2}}\left(r, \vec{n} ; r^{\prime} \vec{n}^{\prime}\right)}{\partial r \partial r^{\prime}}\right|_{r, r^{\prime}=R}=-\frac{\omega}{2 \pi R} \sum_{l=0}^{\infty}(2 l+1) P_{l}\left(\left(\vec{n}, \vec{n}^{\prime}\right)\right) T_{l+1 / 2}(\omega)
$$

where

$$
T_{l+\frac{1}{2}}(\omega)=\frac{1}{2} \frac{\mathrm{d}}{\mathrm{d} \omega} \ln \left(I_{l+\frac{1}{2}}(\omega) K_{l+\frac{1}{2}}(\omega)\right)
$$

is the spectral function in the whole space. Inserting the above type terms in (40) and (41) becomes

$$
\begin{aligned}
\delta \zeta(z)= & -\frac{2 z \sin (\pi z)}{\pi R} \sum_{l=0}^{\infty}(2 l+1) \int_{0}^{\infty} \mathrm{d} \omega \omega^{-2 z} T_{l+\frac{1}{2}}(\omega) \int \mathrm{d} \Omega\left(\frac{\beta}{4 \pi R} f(\vec{n})+\frac{\beta^{2}}{4 \pi R^{2}} f^{2}(\vec{n})\right) \\
& +\frac{8 z \sin (\pi z)}{\pi R} \sum_{l, l^{\prime}=0}^{\infty}\left(l+\frac{1}{2}\right)\left(l^{\prime}+\frac{1}{2}\right) D_{l l^{\prime}} \int_{0}^{\infty} \mathrm{d} \omega \omega^{1-2 z}\left(T_{l+\frac{1}{2}}(\omega)-T_{l^{\prime}+\frac{1}{2}}(\omega)\right)^{2}
\end{aligned}
$$

where

$$
D_{l l^{\prime}}=\frac{\beta^{2}}{16 \pi^{2} R^{2}} \int \mathrm{d} \Omega \int \mathrm{d} \Omega^{\prime} P_{l}\left(\left(\vec{n}, \overrightarrow{n^{\prime}}\right)\right) P_{l^{\prime}}\left(\left(\vec{n}, \overrightarrow{n^{\prime}}\right)\right) f(\vec{n}) f\left(\overrightarrow{n^{\prime}}\right),
$$

$\mathrm{d} \Omega=\mathrm{d} \phi \mathrm{d} \theta \sin \theta$ is the integration measure on the sphere. The first term in (46) at $z=-1 / 2$ is proportional to the vacuum energy of the massless scalar field confined in the spherical region of the radius $R$. Therefore the variation of the vacuum energy is then

$$
\begin{aligned}
\delta E=E_{\mathrm{sph}} \int \mathrm{d} \Omega\left(\frac{\beta}{4 \pi R} f(\vec{n})+\frac{\beta^{2}}{4 \pi R^{2}} f^{2}(\vec{n})\right)+\frac{2}{\pi R} \sum_{l, l^{\prime}=0}^{\infty}\left(l+\frac{1}{2}\right)\left(l^{\prime}+\frac{1}{2}\right) D_{l l^{\prime}} \\
\times \int_{0}^{\infty} \mathrm{d} \omega \omega^{2}\left(T_{l+\frac{1}{2}}(\omega)-T_{l^{\prime}+\frac{1}{2}}(\omega)\right)^{2} .
\end{aligned}
$$

Expansion (B.5) and the addition formula (B.4) imply

$$
D_{l l^{\prime}}=\beta^{2} \sum_{J=0}^{\infty} \sum_{M=-J}^{J} K_{l l^{\prime}}^{J} \frac{\left|f_{M}^{J}\right|^{2}}{(2 J+1)^{2}}
$$


where $K_{l l^{\prime}}^{J}$ are the Klebsch-Gordon coefficients (B.6) and $f_{M}^{J}$ are the expansion coefficients of the deformation function $f$ in the spherical harmonics:

$$
f(\vec{n})=\sum_{J=0}^{\infty} \sum_{M=-J}^{J} f_{M}^{J} Y_{M}^{J}(\vec{n})
$$

Using (49) we may represent (48) as

$$
\delta E=E_{\mathrm{sph}}\left[\frac{\beta}{4 \pi R} \int \mathrm{d} \Omega f(\vec{n})+\frac{\beta^{2}}{4 \pi R^{2}} \int \mathrm{d} \Omega\left(f^{2}(\vec{n})+f(\vec{n}) \hat{H} f(\vec{n})\right)\right]
$$

where $\hat{H}$ is an energy operator

$$
\hat{H} Y_{M}^{J}(\vec{n})=H(J) Y_{M}^{J}(\vec{n})
$$

with $e$-values

$H(J)=\frac{1}{\pi \alpha} \sum_{l=J}^{\infty}\left(l+\frac{1}{2}\right) \sum_{N=-J}^{J} \Lambda_{N}^{J} G_{N}^{J}(\mu) \int_{0}^{\infty} \mathrm{d} \omega \omega^{2}\left(T_{l+\frac{1}{2}}(\omega)-T_{l+N+\frac{1}{2}}(\omega)\right)^{2}$.

Here we used formulae (B.6) and (B.12) for the Klebsch-Gordon coefficients. The evaluation of $H(J)$ which is quite involved, is given (together with all auxiliary formulae) in the appendices. The energy operator $H(J)$ has the following expansion:

$$
H(J)=\alpha_{3} J^{3}+\alpha_{2} J^{2}+\alpha_{1} J+\frac{\alpha_{-1}}{J+\frac{1}{2}}+\frac{\alpha_{-2}}{\left(J+\frac{1}{2}\right)^{2}}+\cdots .
$$

Terms with coefficients $\alpha_{-n}, n=-1,-2,-3, \ldots$ in the above expansion give negligible contributions to the Casimir energy compared to the first three ones

$$
H(J)=-3,03 J^{3}-3,37 J^{2}-0,52 J .
$$

The Casimir energy in the cavity obtained by the small but arbitrary deformations of the spherical region of radius $R$ is, therefore given by

$$
E_{\tilde{S}}=\frac{\alpha}{R}\left(1+\frac{\beta}{4 \pi R} \int \mathrm{d} \Omega f+\frac{\beta^{2}}{4 \pi R^{2}} \int \mathrm{d} \Omega f(\hat{H}+1) f\right) .
$$

\section{Shape dependence of the Casimir energy, discussion}

It is instructive to compare the Casimir energy (56) with the energy in a spherical cavity with equal volume. The volume and the area of the cavity after deformation are (up to $\frac{\beta^{2}}{R^{2}}$ order)

$$
\tilde{V}=\frac{4 \pi}{3} R^{3}\left(1-\frac{3 \beta}{4 \pi R} \int \mathrm{d} \Omega f+\frac{3 \beta^{2}}{4 \pi R^{2}} \int \mathrm{d} \Omega f^{2}\right)
$$

and

$$
\tilde{S}=4 \pi R^{2}\left(1-\frac{\beta}{2 \pi R} \int \mathrm{d} \Omega f+\frac{\beta^{2}}{4 \pi R^{2}} \int \mathrm{d} \Omega f\left(1-\frac{1}{2} \Delta\right) f\right)
$$

where

$$
\Delta=\frac{1}{\sin \theta} \frac{\partial}{\partial \theta} \sin \theta \frac{\partial}{\partial \theta}+\frac{1}{\sin ^{2} \theta} \frac{\partial^{2}}{\partial \phi^{2}}
$$

is the Laplace operator on the sphere. The ratio of the energy (56) and the Casimir energy $E_{0}$ of the sphere with volume (57) is

$$
\left.\frac{E_{\tilde{S}}}{E_{0}}\right|_{\text {eq.vol. }}=1+\frac{\beta^{2}}{4 \pi R^{2}}\left(\int \mathrm{d} \Omega f(2+\hat{H}) f-\frac{1}{2 \pi}\left(\int \mathrm{d} \Omega f\right)^{2}\right) .
$$


This ratio is to be examined by its dependence on the shape of the cavity after deformation. One way may be to study its dependence on the ratio of the surfaces of the deformed and spherical cavities with equal volumes:

$$
\left.\frac{\tilde{S}}{S_{0}}\right|_{\text {eq.vol. }}=1-\frac{\beta^{2}}{4 \pi R^{2}}\left(\int \mathrm{d} \Omega f\left(1+\frac{1}{2} \Delta\right) f-\frac{1}{4 \pi}\left(\int \mathrm{d} \Omega f\right)^{2}\right) .
$$

We then express the energy ratio (60) as

or

$$
\left.\frac{E_{\tilde{S}}}{E_{0}}\right|_{\text {eq.vol. }}=1+\frac{\beta^{2}}{4 \pi R^{2}} \int \mathrm{d} \Omega f(\hat{H}-\Delta) f-2\left(\left.\frac{\tilde{S}}{S_{0}}\right|_{\text {eq.vol. }}-1\right)
$$

$$
\left.\frac{E_{\tilde{S}}}{E_{0}}\right|_{\text {eq.vol. }}=1-2 \frac{\int \mathrm{d} \Omega f\left(1+\frac{1}{2} \hat{H}\right) f-\frac{1}{4 \pi}\left(\int \mathrm{d} \Omega f\right)^{2}}{\int \mathrm{d} \Omega f\left(1+\frac{1}{2} \Delta\right) f-\frac{1}{4 \pi}\left(\int \mathrm{d} \Omega f\right)^{2}}\left(\left.\frac{\tilde{S}}{S_{0}}\right|_{\text {eq.vol. }}-1\right) .
$$

We know from (55) that the operator $\hat{H}$ has negative $e$-values. Thus both of the above relations show that the Casimir energy linearly decreases by the increase of the surface. To have a better feeling of this inverse proportionality let us consider a simple example. Suppose the deformation function is given by

$$
f(\theta, \phi)=P_{l}(\cos \theta)
$$

with $P_{l}$ being the Legendre polynomials. By using (52) and (53), we can write (63) for this specific example as

$$
\left.\frac{E_{\tilde{S}}}{E_{0}}\right|_{\text {eq.vol. }}=1-\Lambda(l)\left(\left.\frac{\tilde{S}}{S_{0}}\right|_{\text {eq.vol. }}-1\right)
$$

where the coefficient $\Lambda$ is given by

$$
\Lambda(l)=2 \frac{2+H(l)}{2-l(l+1)}
$$

or by using (55) can be written as

$$
\Lambda(l)=2 \frac{2-3,03 l^{3}-3,37 l^{2}-0,52 l}{2-l(l+1)} .
$$

Thus for large values of $l$ we can write (65) as

$$
\left.\frac{E_{\tilde{S}}}{E_{0}}\right|_{\text {eq.vol. }}=1-6 l\left(\left.\frac{\tilde{S}}{S_{0}}\right|_{\text {eq.vol. }}-1\right) .
$$

We then can conclude that for less and less smooth deformations we get smaller and smaller Casimir energies.

Before closing the section let us consider the prolate spheroid given by

$$
\frac{x^{2}+y^{2}}{a^{2}}+\frac{z^{2}}{b^{2}}=1, \quad \text { with } \quad \bar{e}^{2}=\frac{a^{2}}{b^{2}}-1 \ll 1
$$

which was previously studied up to order $\bar{e}^{2}$ [19]. The deformation function for this geometry is given by (with $b=R$ )

$$
f=\frac{b}{2 \beta} \bar{e}^{2}\left(\sin ^{2} \theta+\frac{\bar{e}^{2}}{4} \sin ^{4} \theta\right) .
$$

Inserting it into (56) we arrive at the Casimir energy

$$
E=\frac{\alpha}{b}\left(1+\frac{1}{3} \bar{e}^{2}-\frac{3}{5} \bar{e}^{4}\right)=\frac{1}{2 b}\left(0,006+0,002 \bar{e}^{2}\right)-\frac{0,002}{b} \bar{e}^{4}
$$

which is in agreement with the result of [19]. 


\section{Acknowledgments}

The authors thank the Turkish Academy of Science (TUBA) for its support, D A Demir and T O Turgut for discussions and to P Talazan for helping in 'Mathematica'.

\section{Appendix A. The calculation of the vacuum energy}

Using the uniform expansion formula (16) for the spectral function (45) we represent the energy operator (52) (with $\mu=l+1 / 2, \omega=\mu x$ and $\varepsilon=\frac{N}{\mu}$ ) as

$H(J)=\frac{1}{\pi \alpha} \sum_{n, m=0}^{\infty} \sum_{l=J}^{\infty} \mu^{2-2 m-2 n} \sum_{N=-J}^{J} \Lambda_{N}^{J} D_{l}(N, J) \int_{0}^{\infty} \mathrm{d} x x^{2} Y_{2 n}(x, \varepsilon) Y_{2 m}(x, \varepsilon)$

where

$$
Y_{n}(x, \varepsilon)=\frac{\mathrm{d}}{\mathrm{d} x}\left(\frac{X_{n}(t(\varepsilon))}{(1+\varepsilon)^{n}}-X_{n}(t(0))\right)
$$

and

$$
t(\varepsilon)=\frac{1+\varepsilon}{\sqrt{(1+\varepsilon)^{2}+x^{2}}} .
$$

After the change of variables $2 n=s+t, 2 m=s-t$ we have

$$
H(J)=\sum_{s=0}^{\infty} H^{s}(J)
$$

where

$$
H^{s}(J)=\frac{1}{\pi \alpha} \sum_{l=J}^{\infty} \mu^{3-2 s} \sum_{N=-J}^{J} \Lambda_{N}^{J} G_{N}^{J}(\mu) \int_{0}^{\infty} \mathrm{d} x x^{2} F_{s}(x, \varepsilon)
$$

and

$$
F_{s}(x, \varepsilon)=\sum_{t=-s}^{s} Y_{s+t}(x, \varepsilon) Y_{s-t}(x, \varepsilon) .
$$

By using the Taylor expansion at $\varepsilon=0$

$$
F_{s}(x, \varepsilon)=\sum_{k=0}^{\infty} \frac{\varepsilon^{n}}{n !} F_{s}^{(n)} x
$$

and the asymptotic expansion (B.13) we arrive at

$$
H^{s}(J)=\sum_{\tau=0}^{\infty} b_{\tau} \zeta(2 \tau+2 s-2, J)
$$

where

$$
\zeta(z, J)=\sum_{k=0}^{\infty} \frac{1}{\left(J+\frac{1}{2}+k\right)^{z}}
$$

is the Riemann zeta function

$$
b_{\tau}=\frac{1}{\pi \alpha} \sum_{p=-\tau}^{\tau} \frac{\left\langle Z_{\tau+p} N^{\tau-p}\right\rangle}{(\tau-p) !} \int_{0}^{\infty} \mathrm{d} x x^{2} F_{s}^{(\tau-p)}(x)
$$

10 
and

$$
\langle f(N)\rangle=\sum_{N=-J}^{J} \Lambda_{N}^{J} f(N) .
$$

For large $J$ we have

$$
\zeta(2 \tau+2 s-2, J) \simeq J^{3-2 \tau-2 s}
$$

and

$$
\left\langle Z_{\tau+p} N^{\tau-p}\right\rangle \simeq J^{2 \tau}
$$

These asymptotic expressions imply

$$
H^{s}(J) \simeq J^{3-2 s}, \quad J \gg 1 .
$$

Thus $H^{0}(J)$ and $H^{1}(J)$ give the main contributions to the energy operator $H(J)$. Using the summation formulae (B.18) we get

$$
H^{0}(J)=\frac{1}{128 \alpha}\left(J(J+1) \zeta(0, J)-\frac{J(J+2)\left(J^{2}-1\right)}{8} \zeta(2, J)\right)
$$

and

$$
H^{1}(J)=-\frac{9}{2048 \alpha}\left(\frac{J(J+1)}{2} \zeta(2, J)+\frac{99}{16 \cdot 24}\left(J+\frac{1}{2}\right)^{4} \zeta(4, J)\right)
$$

from which by the virtue of

$$
\begin{aligned}
& \zeta(2, J) \approx \frac{1}{J+1 / 2}+\frac{1}{2(J+1 / 2)^{2}}+\frac{1}{6(J+1 / 2)^{3}}+o(J) \\
& \zeta(4, J) \approx \frac{1}{3(J+1 / 2)^{3}}+\frac{1}{2(J+1 / 2)^{4}}+o(J)
\end{aligned}
$$

we read

$$
\alpha_{3}=-\frac{9}{1024 \alpha}, \quad \alpha_{2}=-\frac{10}{1024 \alpha}, \quad \alpha_{1}=-\frac{1193}{1024 \cdot 768 \alpha}
$$

\section{Appendix B. The Klebsch-Gordon coefficients}

The spherical harmonics are

$$
Y_{m}^{l}(\theta, \phi)= \begin{cases}2 P_{l}^{m}(\cos \theta) \cos m \phi, & m=1,2, \ldots, l \\ P_{l}(\cos \theta), & m=0 \\ 2 P_{l}^{|m|}(\cos \theta) \sin |m| \phi, & m=-1,-2, \ldots,-l\end{cases}
$$

where $P_{l}(x)$ is the Legendre function and

$$
P_{l}^{m}(x)=\sqrt{\frac{l-m) !}{(l+m) !}}\left(1-x^{2}\right)^{\frac{m}{2}} \frac{\mathrm{d}^{m}}{\mathrm{~d} x^{m}} P_{l}(x)
$$

is the associated Legendre function. We use the notation $Y_{m}^{l}(\theta, \phi)=Y_{m}^{l}(\vec{n})$ where

$$
\vec{n}=(\cos \theta, \sin \theta \sin \phi, \sin \theta \cos \phi)
$$

is the unit vector on the sphere. 
The addition formula for the spherical harmonics is

$$
P_{l}\left(\left(\vec{n}, \vec{n}^{\prime}\right)\right)=\sum_{m=-l}^{l} Y_{m}^{l}(\hat{n}) Y_{m}^{l}\left(\hat{n}^{\prime}\right)
$$

where the argument of the Legendre function is the scalar product of the unit vectors $\vec{n}$ and $\vec{n}^{\prime}$.

The Klebsch-Gordon coefficients $K_{l l+N}^{J}$ defined by the expansion

$$
P_{l}(x) P_{l^{\prime}}(x)=\sum_{J=\left|l-l^{\prime}\right|}^{l+l^{\prime}} K_{l l^{\prime}}^{J} P_{J}(x)
$$

are [20]

$$
K_{l l+N}^{J}=\left(J+\frac{1}{2}\right) \Lambda_{N}^{J} F_{l}(N, J)
$$

where $(\mu=l+1 / 2)$

$$
F_{l}(N, J)=\frac{1}{\mu+\frac{N+J}{2}} \frac{\Gamma\left(\mu+\frac{N-J}{2}\right) \Gamma\left(\mu+\frac{N+J}{2}+\frac{1}{2}\right)}{\Gamma\left(\mu+\frac{N-J}{2}+\frac{1}{2}\right) \Gamma\left(\mu+\frac{N+J}{2}\right)}
$$

and

$$
\Lambda_{N}^{J}=\frac{1}{\pi} \frac{\Gamma\left(\frac{J-N+1}{2}\right) \Gamma\left(\frac{J+N+1}{2}\right)}{\Gamma\left(\frac{J-N+2}{2}\right) \Gamma\left(\frac{J+N+2}{2}\right)} .
$$

These coefficients are nonzero if $|N| \leqslant J \leqslant 2 l+N$ and $N+J$ is even number.

The formula

$$
\ln \Gamma(z)=z \ln z-z-\frac{1}{2} \ln z+\ln \sqrt{2 \pi}+\sum_{k=1}^{n-1} \frac{B_{2 k}}{2 k(2 k-1) z^{2 k-1}}
$$

implies

$$
\ln \frac{\Gamma\left(z+\frac{1}{2}\right.}{\Gamma(z)}=\frac{1}{2} \ln z-\frac{1}{8 z}+\frac{1}{192 z^{3}}
$$

up to the third order in $\frac{1}{z}$. The last formula allows us to get the asymptotic expansion of the functions $F_{l}(N, J)$. Up to the third order in $\frac{1}{\mu}$ for large values of $\mu$ we have

$$
F_{l}(N, J)=\frac{1}{\sqrt{\left(\mu+\frac{N-J}{2}\right)\left(\mu+\frac{N+J}{2}\right)}}\left(1+\frac{J}{8 \mu^{2}}-\frac{N J}{8 \mu^{3}}\right) .
$$

For the function

$$
G_{N}^{J}(\mu)=(\mu+N) F_{l}(N, J)
$$

we get the following asymptotic expansion:

$$
G_{N}^{J}(\mu)=1+\frac{Z_{1}}{\mu}+\frac{Z_{2}}{\mu^{2}}+\frac{Z_{3}}{\mu^{3}}+\cdots
$$

where

$$
Z_{1}=\frac{N}{2}, \quad Z_{2}=\frac{J(J+1)-2 N^{2}}{8}, \quad Z_{3}=N \frac{2 N^{2}-J(J+1)}{16} .
$$

Let $J$ be even. Putting $J=2 j$ and $N=2 n$ we have

$$
\sum_{N=-J}^{J} \Lambda_{N}^{J} \mathrm{e}^{\mathrm{i} N \theta}=\sum_{n=-j}^{j} \frac{1}{\pi} \frac{\Gamma\left(j-n+\frac{1}{2}\right) \Gamma\left(j+n+\frac{1}{2}\right)}{(j-n) !(j+n) !} \mathrm{e}^{\mathrm{i} 2 n \theta} .
$$


In the new variable $m=n+j$ we have

$$
\sum_{N=-J}^{J} \Lambda_{N}^{J} \mathrm{e}^{\mathrm{i} N \theta}=\sum_{m=0}^{2 j} \frac{1}{\pi} \frac{\Gamma\left(m+\frac{1}{2} \Gamma\left(2 j-m+\frac{1}{2}\right)\right.}{m !(2 j-m) !} \mathrm{e}^{\mathrm{i}(2 m-2 j) \theta}
$$

which is exactly the series representation for zonal spherical functions $Y_{0}^{2 j}(\cos \theta)$

$$
\sum_{N=-J}^{J} \Lambda_{N}^{J} \mathrm{e}^{\mathrm{i} N \theta}=Y_{0}^{J}(\cos \theta)
$$

The same formula is true for odd $J$. For example, we have

$$
\begin{aligned}
& \sum_{N=-J}^{J} \Lambda_{N}^{J}=1, \\
& \sum_{N=-J}^{J} \Lambda_{N}^{J} N^{2}=\frac{J(J+1)}{2}, \\
& \sum_{N=-J}^{J} \Lambda_{N}^{J} N^{4}=\frac{3 J^{3}(J+2)+J(J-2)}{8} .
\end{aligned}
$$

\section{References}

[1] See, for example, the monograph by Mostepanenko V M and Trunov N N 1997 The Casimir Effect and its Applications (New York: Oxford University Press) (references therein)

Balian R B and Bloch C 1970 Ann. Phys., NY 60401

Ambjorn J and Wolfram S 1983 Ann. Phys. 1471

Edery A 2006 J. Phys. A: Math. Gen. 39685

[2] Boyer T H 1968 Phys. Rev. 1741764

Davies B 1972 J. Math. Phys. 131324

Milton K A, DeRaad L L and Schwinger J S 1978 Ann. Phys. 115388

Lambiase G, Nesterenko V V and Bordag M 1999 J. Math. Phys. 406254

Esposito G, Kamenshchik A Y and Kirsten K 2000 Phys. Rev. D 62085027

[3] Barton G 2004 J. Phys. A: Math. Gen. 371011

ReDood L L (Jr) and Milton K A 1981 Ann. Phys. 136229

Gosdzinsky P and Romeo A 1998 Phys. Lett. B 441265

Lifshitz E M 1956 Zh. Eksp. Teor. Fiz. 2994

Dowker J S and Critchley R 1976 J. Phys. A: Math. Gen. 9535

[4] Ahmedov H and Duru I H 2005 J. Math. Phys. 46022303

Ahmedov H and Duru I H 2005 J. Math. Phys. 46022304

[5] Derjaguin B 1934 Kolloid Z. 69155

[6] See for a recent discussion, Sernelius Bo E and Roman-Velasques C E 2008 arXiv:quant-ph/08071626

[7] See, for example, Emig T, Hanke A, Golestanian R and Kadar M 2003 Phys. Rev. A 64022114

[8] Balian R and Duplantier B 1978 Ann. Phys. 112165

[9] Fulling S A 2007 Sigma 3165

[10] See for a general discuasion, Balian R and Duplantier B 2004 arXiv:quant-ph/0408124v1

[11] Golestanian R and Kadar M 1998 Phys. Rev. A 581713

[12] Minakshisundram S and Pleijel A 1949 Can. J. Math. 1142

Minakshisundram S J 1953 Indian Math. Soc. 17158

Gilkey P G 1995 Invariant Theory, The Heat Kernel Equation and the Atiah-Singer Index Theorem 2nd edn (Boca Raton, FL: CTC Press)

Vasilevich D V 2003 Phys. Rep. 388279

[13] Borgad M, Mohideen U and Mostepanenko V M 2001 Phys. Rep. 3531

[14] Kirsten K 2002 Spectral Functions in Mathematics and Physics (Boca Raton: Chapman and Hall)

[15] See, for example, Abramowitc M and Stegun I 1972 Habdbook of Mathematical Functions (New York: Dower) 
[16] Cognola G, Elizade E and Kirsten K 2001 J. Phys. A: Math. Gen. 34 7311-27

[17] Gradshtejn S and Ryzhik I M 1965 Tables of Integrals, Series and Products (New York: Academic)

[18] Vladimirov V S 1976 Equations of Mathematical Physics 3rd edn (Moscow: Nauka)

Vladimirov V S 1971 Equations of Mathematical Physics 1st edn (New York: Marcel Dekker) (Engl. Transl.)

[19] Kitson A R and Signal A L 2006 J. Phys. A: Math. Gen. 396473

[20] Vilenkin N and Klimyk A 1993 Representations of Lie Groups and Special Functions, Mathematics and its Application vol 1 (Dordrecht: Kluwer) 